\title{
DUALITY IN CONVEX OPTIMIZATION
}

\author{
CHRISTOPHER J. HILLAR
}

\section{INTRODUCTION}

Consider the problem [1]:

$$
\begin{gathered}
\text { minimize } f_{0}(x) \\
\text { subject to } f_{i}(x) \leq 0 \\
h_{i}(x)=0 .
\end{gathered}
$$

where $f_{0}(x)$ is a convex function and the $h_{i}(x)$ are affine. Set $\mathcal{D}$ to be the intersection of the domains of the $f_{i}$. We set $p^{*}$ to be the solution to this minimization problem.

Define the Lagrangian associtated to the problem to be

$$
L(x, \lambda, \nu)=f_{0}(x)+\sum_{i=1}^{m} \lambda_{i} f_{i}(x)+\sum_{i=1}^{p} \nu_{i} h_{i}(x) .
$$

The $\lambda$ and $\nu$ are called dual variables.

We would like to minimize this function over the domain:

$$
g(\lambda, \nu):=\inf _{x \in \mathcal{D}} L(x, \lambda, \nu) .
$$

We define the domain of $g$ to be those points for which $g$ is not $-\infty$ :

$$
\operatorname{dom} g=\{(\lambda, \nu): g(\lambda, \nu)>-\infty\} .
$$

Theorem 1.1. When $\lambda \geq 0$, we have

$$
g(\lambda, \nu) \leq p^{*} .
$$

Proof. Clearly, $g(\lambda, \nu) \leq L(x, \lambda, \nu)$ for all $x \in \mathcal{D}$. In particular, for feasible $\tilde{x}$, we have

$$
g(\lambda, \nu) \leq f_{0}(\tilde{x}),
$$

since $\lambda$ are all nonnegative and $h_{i}(\tilde{x})=0$.

It follows that we can obtain a lower bound on $p^{*}$ by solving the following optimization problem, called the Lagrange dual problem associated to the original optimization problem:

$$
\begin{aligned}
& \text { maximize } g(\lambda, \nu) \\
& \text { subject to } \lambda \geq 0 .
\end{aligned}
$$

We will denote the answer to this problem as $d^{*}$. Of course, we have

$$
d^{*} \leq p^{*},
$$


a property we call weak duality. This turns out to be a convex optimization problem since the objective to be maximized is concave and the constraint is convex. This concavity can be proved directly using the fact that the infimum of a sum is greater than or equal to the sum of the infimums.

\section{Strong Duality}

Under mild conditions, we show that both optimization problems have the same solution; that is, $d^{*}=p^{*}$.

To begin, we define the set

$$
\mathcal{A}=\left\{(u, v, t): \exists x \in \mathcal{D}, f_{i}(x) \leq u_{i}, i=1, \ldots, m, h_{i}(x)=v_{i}, i=1, \ldots, p, f_{0}(x) \leq t\right\},
$$

which is convex since each of the $f_{i}(x)$ are convex functions.

The optimal solution to our original problem is:

$$
p^{*}=\inf \{t:(0,0, t) \in \mathcal{A}\} .
$$

Next, notice that if $\lambda \geq 0$,

$$
g(\lambda, \nu)=\inf \left\{(u, v, t)(\lambda, \nu, 1)^{T}:(u, v, t) \in \mathcal{A}\right\} .
$$

If $\lambda \geq 0$ and $\nu$ are given and $g(\lambda, \nu)$ is finite, then

$$
(u, v, t)(\lambda, \nu, 1)^{T} \geq g(\lambda, \nu)
$$

defines a (nonvertical) supporting hyperplane (really, half-space) to $\mathcal{A}$.

In particular, since $\left(0,0, p^{*}\right) \in \mathrm{bd} \mathcal{A}$, we have

$$
p^{*}=(\lambda, \nu, 1)^{T}\left(0,0, p^{*}\right) \geq g(\lambda, \nu) .
$$

To rephrase in this language, Strong duality holds if and only if we have equality in equation (2.1).

\section{Slater's COnstraint qualification}

Definition 3.1 (Slater's Condition). There exists an $\tilde{x} \in \operatorname{relint} \mathcal{D}$ with $f_{i}(\tilde{x})<0$ for $i=1, \ldots, m$ and $A \tilde{x}=b$.

In other words, there exists a strictly feasible point.

Theorem 3.2. Slater's condition implies strong duality.

Proof sketch. Assume for simplicity that relint $\mathcal{D}=\operatorname{int} \mathcal{D}$ and that $A$ has full rank $p$. Consider the following (convex) set

$$
\mathcal{B}=\left\{(0,0, s) \in \mathbb{R}^{m} \times \mathbb{R}^{p} \times R: s<p^{*}\right\},
$$

which is obviously disjoint from $\mathcal{A}$.

By the separating hyperplane theorem, there exists $(\tilde{\lambda}, \tilde{\nu}, \mu) \neq 0$ and $\alpha$ such that

$$
(u, v, t) \in \mathcal{A} \Rightarrow u \tilde{\lambda}^{T}+v \tilde{\nu}^{T}+t \mu \geq \alpha,
$$

and

$$
(u, v, t) \in \mathcal{B} \Rightarrow u \tilde{\lambda}^{T}+v \tilde{\nu}^{T}+t \mu \leq \alpha,
$$

This implies that $\tilde{\lambda} \geq 0$, by the first equation (since $\mathcal{A}$ is closed under $u$ getting larger), and $\mu \geq 0$, by the second, which says that $\mu t \leq \alpha$ for all $t<p^{*}$ and thus $\mu p^{*} \leq \alpha$. 
For any $x \in \mathcal{D}$, we have

$$
\left(f_{1}(x), \ldots, f_{m}(x), h_{1}(x), \ldots, h_{p}(x), f_{0}(x)\right) \in \mathcal{A},
$$

and therefore by the first inequality, it follows that

$$
\sum_{i=1}^{m} \tilde{\lambda}_{i} f_{i}(x)+\tilde{\nu}(A x-b)+\mu f_{0}(x) \geq \alpha \geq \mu p^{*} .
$$

We proceed in two cases.

Case 1: $\mu>0$. Dividing (3.1) by $\mu$, we obtain

$$
L(x, \tilde{\lambda} / \mu, \tilde{\nu} / \mu) \geq p^{*},
$$

for all $x \in \mathcal{D}$. Thus, minimizing over $x$, it follows that $g(\lambda, \nu) \geq p^{*}$ where $\lambda=\tilde{\lambda} / \mu$ and $\nu=\tilde{\nu} / \mu$. By weak duality, we have $g(\lambda, \nu)=p^{*}$.

Case 1: $\mu=0$. Using (3.1), it follows that for $\tilde{x}$ satisfying Slater's condition, we have

$$
\sum_{i=1}^{m} \tilde{\lambda}_{i} f_{i}(\tilde{x}) \geq 0
$$

and therefore $\tilde{\lambda}=0$ since all $f_{i}(\tilde{x})<0$ and $\tilde{\lambda} \geq 0$. From $(\tilde{\lambda}, \tilde{\nu}, \mu) \neq 0$ and $\tilde{\lambda}=\mu=0$, we conclude that $\tilde{\nu} \neq 0$. Thus, (3.1) implies that

$$
\tilde{\nu}(A x-b) \geq 0 .
$$

By assumption, $\tilde{\nu}(A \tilde{x}-b)=0$, and since $\tilde{x} \in \operatorname{int} \mathcal{D}$, it follows that there exists a perturbation $x \in \mathcal{D}$ such that $\tilde{\nu}(A x-b)<0$ unless $\tilde{\nu} A=0$. This contradicts the fact that $\operatorname{rank}(A)=p$. 


\section{REFERENCES}

[1] S. Boyd, L. Vandenberghe, Convex Optimization. 